C.C. Chen · C.M. Chen · F.C. Hsu $\cdot$ C.J. Wang

J.T. Yang $\cdot$ Y.Y. Kao

\title{
The pachytene chromosomes of maize as revealed by fluorescence in situ hybridization with repetitive DNA sequences
}

Received: 29 June 1999 / Accepted: 10 November 1999

\begin{abstract}
A repetitive DNA sequence, ZmCR2.6c, was isolated from maize based on centromeric sequence CCS1 of the wild grass Brachypodium sylvaticum. $\mathrm{ZmCR} 2.6 \mathrm{c}$ is $309 \mathrm{bp}$ in length and shares $65 \%$ homology to bases 421-721 of the sorghum centromeric sequence pSau3A9. Fluorescence in situ hybridization (FISH) localized $\mathrm{ZmCR} 2.6 \mathrm{c}$ to the primary constrictions of pachytene bivalents and to the stretched regions of MI/AI chromosomes, indicating that ZmCR2.6c is an important part of the centromere. Based on measurements of chromosome lengths and the positions of FISH signals of several cells, a pachytene karyotype was constructed for maize inbred line KYS. The karyotype agrees well with those derived from traditional analyses. Four classes of tandemly repeated sequences were mapped to the karyotype by FISH. Repeats 180 bp long are present in cytologically detectable knobs on $5 \mathrm{~L}, 6 \mathrm{~S}, 6 \mathrm{~L}, 7 \mathrm{~L}$, and $9 \mathrm{~S}$, as well as at the termini and in the interstitial regions of many chromosomes not reported previously. A most interesting finding is the presence of 180 -bp repeats in the NOR-secondary constriction. TR-1 elements co-exist with 180-bp repeats in the knob on $6 \mathrm{~S}$ and form alone a small cluster in $4 \mathrm{~L}$. $26 \mathrm{~S}$ and $5 \mathrm{~S}$ rRNA genes are located in the NOR and at 2L.88, respectively. The combination of chromosome length, centromere position, and distribution of the tandem repeats allows all chromosomes to be identified unambiguously. The results presented form an important basis for using FISH for physical mapping and for investigating genome organization in maize.
\end{abstract}

Communicated by B.S. Gill

C.C. Chen ( ) F.C. Hsu · C.J. Wang · J.T. Yang · Y.Y. Kao Department of Botany, National Taiwan University, Taipei, Taiwan, Republic of China

e-mail: chencc@ccms.ntu.edu.tw

Fax: +886-2-2391-8940

C.M. Chen

Institute of Botany, Academia Sinica, Taipei, Taiwan, Republic of China
Key words Zea mays . Pachytene chromosome . Karyotype $\cdot$ Repetitive DNA sequence $\cdot$ Fluorescence in situ hybridization

\section{Introduction}

Fluorescence in situ hybridization (FISH) is a most direct approach for physically mapping cloned genes and DNA fragments. In humans, single-copy sequences as small as $0.9 \mathrm{~kb}$ in length have been mapped, and two sequences separated by not more than $1 \mathrm{Mb}$ have been ordered on mitotic metaphase chromosomes by FISH (Viegas-Péquignot et al. 1991; Trask et al. 1991). In plants, however, the sensitivity and resolution of metaphase FISH have been low because of difficulties in chromosome preparation and the pronounced condensation of metaphase chromosomes (Jiang and Gill 1994).

Recently, several groups of investigators discussed the advantages of using meiotic pachytene chromosomes for physical mapping in plants (Xu and Earle 1996a,b; Zhong et al. 1996a,b; Armstrong et al. 1998; Chen et al. 1998; Fransz et al. 1998). First, pachytene chromosomes are more extended and possess many landmarks not recognizable in mitotic metaphase chromosomes. Second, the walls of pollen mother cells (PMCs) can easily be broken by enzyme treatment, thus facilitating chromosome spreading and probe penetration. Third, the developmental stage of PMCs in an anther, or in anthers of a flower bud, is synchronous. Once the right stage is found, there will be sufficient material for analysis. Fourth, a pachytene bivalent consists of two homologous chromosomes, so hybridization signals on pachytene chromosomes should be stronger than those on unpaired somatic chromosomes.

Among the many economically important plants, maize is one of the most suitable materials for development of a physical map by FISH. The pachytene chromosomes of maize can be obtained and identified easily by the acetocarmine squash technique (Dempsey 1994) and have been widely used for cytogenetic analysis 
(Carlson 1988). Other advantages of maize are the availability of genetic maps based on phenotypic and molecular markers (Neuffer et al. 1997) and the availability of an YAC library (Edwards et al. 1992).

The most useful landmark for chromosome identification is the centromere. Unfortunately, the centromeres of maize pachytene chromosomes are not easily recognized when stained with fluorochromes such as propidium iodide (PI) and 4, 6-diamidine-2-phenylindole dihydrochloride (DAPI). This is particularly true for the centromeres of chromosomes $1,3,4,5,6$, and 8 . A breakthrough occurred when centromeric sequences of several cereal species were isolated and characterized (AragónAlcaide et al. 1996; Jiang et al. 1996; Dong et al. 1998; Ananiev et al. 1998c; Miller et al. 1998; Presting et al. 1998; Nonomura and Kurata 1999) and one of these, the sorghum pSau3A9 sequence, was successfully mapped to the centromeres of maize pachytene chromosomes (Chen et al. 1998).

Knobs are heterochromatic regions on maize chromosomes and are most conspicuous at prophase of the first meiotic division. The size, number, and chromosomal distribution of knobs vary among strains but are constant within strains (McClintock et al. 1981). Recently, Ananiev et al. (1998a,b) found that the molecular structure of knobs is complex, consisting of two tandem repeats, the 180-bp element discovered by Peacock et al. (1981) and a new 350-bp element (TR-1), and various retrotransposons. The two tandem repeats may be present in the same knob, in different knobs, or in regions where knob heterochromatin is not apparent (Ananiev et al. 1998b). Knobs are excellent landmarks on maize pachytene chromosomes.

In investigation reported here we isolated a centromeric sequence and the 180-bp and TR-1 repeats from maize genomic DNA based on previously published sequences and used these, together with Nicotiana plumbaginifolia 26S rDNA and rice 5S rDNA, as probes to hybridize pachytene chromosomes of maize inbred line KYS. From the distribution of these sequences we constructed a karyotype. The technique and results presented form an important basis for using FISH for physical mapping and for investigating chromosome orientation and pairing during meiosis.

\section{Materials and methods}

Plant material and chromosome preparation

Zea mays L. inbred line KYS $(2 n=20)$, obtained from the Maize Genetics Cooperation Stock Center, University of Illinois, USA, and from Prof. D.B. Walden, Department of Plant Science, University of Western Ontario, Canada, was used in all experiments. Meiotic chromosome spreads for FISH were prepared according to Chen et al. (1998).

Cloning of centromeric and knob-associated sequences

Centromeric sequences

Maize genomic DNA was isolated as described by Suen et al. (1997). Centromeric sequences were amplified by polymerase chain reaction (PCR) with maize genomic DNA and oligonucleotide 5'GGTGCCCGATCTTTCGATGAGA3'. The oligonucleotide spans positions 236-214 in centromeric sequence CCS1 of the wild grass Brachypodium sylvaticum and was designated primer D by Aragón-Alcaide et al. (1996). The PCR products were polished by T4 polymerase and cloned into pUC18 (Sambrook et al. 1989). The cloned PCR products were screened for centromeric sequences by FISH to maize pachytene chromosomes. Positive clones were sequenced using an Applied Biosystem ABI377 DNA sequencer. Homology searches were made against sequences in the nucleic acid database of Genbank using BLASTN.

\section{0-bp repeats}

Genomic DNA of maize was digested with HaeIII (Peacock et al. 1981) and electrophoresed on a $1.5 \%$ agarose gel. DNA fragments corresponding to the monomer and dimer of the 180-bp repeat were recovered, cloned into pUC18, and screened by colony and Southern blot hybridizations using oligonucleotide 5'AAGGAAACATATGTGGGGAGAGGTGTA3', a conserved region in the repeat (Dennis and Peacock 1984), as a probe. Some positive clones were sequenced.

\section{TR-1 (350-bp) repeats}

Genomic DNA of maize was digested with EcoRI (Ananiev et al. 1998b) and electrophoresed on a $1.5 \%$ agarose gel. DNA fragments corresponding to the monomer of the TR-1 repeat were recovered, cloned into pUC18 and screened by colony and Southern blot hybridizations using oligonucleotide 5'TTAGAGTACAAC TAGTGGATGAAG3' (Ananiev et al. 1998b) as a probe. Some positive clones were sequenced.

Source and labeling of probes

Five repetitive sequences were used as probes. Centromeric sequence ZmCR2.6c, a dimer of the 180-bp repeat, ZmKR180-2, and a monomer of the TR-1 repeat, ZmKR350-1, were obtained in the present study. pRTy5S, a 3-kb tandem array of rice 5S rDNA cloned into pTZ19R, was provided by Dr. H.K. Wu, Institute of Botany, Academia Sinica (Chung et al. 1993). pNP26S is a 1-kb EcoRI fragment from Nicotiana plumbaginifolia 26S rDNA cloned into pBluescript (Y.Y. Kao unpublished data). Probes were labeled with digoxigenin-11-dUTP or biotin-16-dUTP by nick translation or PCR according to the protocols of labeling kits provided by the manufacturer (Boehringer Mannhein).

\section{Fluorescence in situ hybridization}

The hybridization mixture consisted of $40-50 \%$ formamide (depending on the homology between probe and target sequences), $2 \times$ standard saline citrate (SSC), $10 \%$ dextran sulfate, $0.1 \%$ sodium dodecyl sulfate (SDS), $5 \mathrm{ng} / \mu \mathrm{l}$ probe DNA, and $1 \mu \mathrm{g} / \mu \mathrm{l}$ herring sperm DNA. Hybridization was carried out at $37^{\circ} \mathrm{C}$ for $20 \mathrm{~h}$, followed by washes in $20 \%$ formamide, $0.2-2 \times$ SSC (depending on the homology between probe and target sequences) at $42^{\circ} \mathrm{C}$ for $10 \mathrm{~min}$, in $2 \times \mathrm{SSC}$ at $42^{\circ} \mathrm{C}$ for $10 \mathrm{~min}$, and in $2 \times \mathrm{SSC}$ at room temperature for $3 \times 5 \mathrm{~min}$. To investigate the relative chromosomal locations of two repetitive sequences, we hybridized the probes to pachytene spreads either simultaneously or sequentially as described by Heslop-Harrison et al. (1992). Digoxigenin-labeled probes were detected with fluorescein-conjugated antibodies (Boehringer Mannhein), and signals were amplified with fluorescein-conjugated anti-sheep IgG (Vector). Biotin-labeled probes were detected with avidin-Texas Red (Vector). Chromosomes were counterstained with PI or DAPI. Slides were visualized under an Olympus AX70 fluorescence microscope with appropriate filter sets, and the images were photographed on Fujicolor Supera 200 ASA print film. For karyotype construction, images on the negatives were projected and measured on white papers at a magnification of $6,000 \times$. 


\section{Results}

Isolation and characterization of a centromeric sequence

PCR with maize genomic DNA and primer D of AragónAlcaide et al. (1996) yielded six major fragments of $0.9 \mathrm{~kb}, 1.0 \mathrm{~kb}, 1.1 \mathrm{~kb}, 1.5 \mathrm{~kb}, 1.8 \mathrm{~kb}$, and $2.1 \mathrm{~kb}$. These fragments were cloned and tested for the presence of the centromeric sequence by FISH to maize pachytene chromosomes. Among these clones, pZmCR2.6 (1.8 kb) hybridized the strongest to primary constrictions (data not shown). Digestion of pZmCR2.6 with restriction enzymes KpnI and HindIII yielded three subfragments of $1.0 \mathrm{~kb}, 0.5 \mathrm{~kb}$, and $0.3 \mathrm{~kb}$. Only subclone pZmCR2.6c $(0.3 \mathrm{~kb})$ was successfully sequenced (EMBL accession number Y18897). ZmCR2.6c is $65 \%$ identical to bases 421-721 of the sorghum pSau3A9 sequence and is $33 \%$ identical to bases 415-763 of a barley 809-bp sequence, the putative LTR/integrase coding regions of Ty3/gypsytype retrotransposons (Miller et al. 1998; Presting et al. 1998).

FISH of ZmCR2.6c to meiotic chromosomes of KYS showed that hybridization signals occurred exclusively in the primary constrictions of pachytene bivalents (Fig. 1A,B) and in the stretched regions of metaphase I (MI) and anaphase I (AI) chromosomes (Fig. 1C). We also found that signals in centromeres of the ten pachytene chromosomes could be classified into two types: those in chromosomes 2, 7, 9, and 10 were weaker, more diffused, and located in the center of the centromere, while those in the remaining chromosomes were stronger, more condensed, and embedded in the pericentric heterochromatin (Fig. 1A). Giemsa staining of the same cells after FISH showed that centromeres of the first type were larger in size, lighter in color - almost appear as gaps - and more easily identified than those of the second type (Fig. 1D).

Pachytene karyotype revealed by FISH with a centromere-specific sequence

The pachytene chromosomes could be easily traced and their centromeres were clearly visible in PMCs hybridized with $\mathrm{ZmCR} 2.6 \mathrm{c}$ (Fig. 1A, B). Although some chromosome regions were stretched somewhat, the individual chromosomes in many cells could still be identified with the aid of other cytological markers such as knobs, nucleolar organizer region (NOR) and satellite.

Seven good-quality pachytene spreads (e.g., Fig. 1A, B) were selected for measurements of the karyotype. The chromosome lengths, expressed as percentages of total complement length (\% TCL), and arm ratios were calculated. In the measurements, the heterochromatic NOR was included as a portion of the short arm of chromosome 6 , but the secondary constriction and the satellite were excluded, in accordance with the accepted practice in karyotyping with somatic metaphase chromosomes. As shown in Table 1, results from FISH are consistent with those obtained from traditional analyses. The only major discrepancy among investigations is the arm ratio of chromosome 6 (see Discussion).

\section{Localization of 180-bp repeats}

Many clones containing the 180 -bp repeat were isolated but only four were sequenced. Of these, one is a monomer and one is a dimer of the 180-bp repeat; the remaining two are 202-bp related sequences. Homology among these sequences and those reported by Peacock et al. (1981), Dennis and Peacock (1984) and Ananiev et al. (1998a) is 82-97\%.

FISH of the 180-bp repeat to pachytene chromosomes revealed that in addition to knobs on $5 \mathrm{~L}, 6 \mathrm{~S}, 6 \mathrm{~L}, 7 \mathrm{~L}$, and 9S, which were heavily labeled as expected from the results of Peacock et al. (1981), medium and weak hybridization signals were detected at many chromosomal sites where heterochromatic knobs were not apparent (Fig. 1E). In order to determine the precise locations of the medium and weak signals, we first hybridized pachytene chromosomes with ZmCR2.6c to localize centromeres (Fig. 1B), then we washed slides with $4 \times \mathrm{SSC} / \mathrm{Tween}$ 20 and reprobed chromosome preparations with the 180-bp repeat (Fig. 1F). In this way, we were able to show that the termini of $1 \mathrm{~S}, 2 \mathrm{~S}, 3 \mathrm{~S}, 6 \mathrm{~L}$ and $8 \mathrm{~S}$, the secondary constriction on $6 \mathrm{~S}$, and sites at 1L.51, 4L.42, 4L.55, and 5S.34 all contain 180-bp repeats (cf. Fig. 1B, E and F; Fig. 2).

To understand the relative position of rDNA and the 180-bp repeat in the secondary constriction, we hybridized pachytene chromosomes simultaneously with $N$. plumbaginifolia $26 \mathrm{~S}$ rDNA and $180-\mathrm{bp}$ repeats. At late

Fig. 1A-J Distribution of repetitive DNA sequences on meiotic chromosomes of maize inbred line KYS. A,B Pachytene chromosomes (red) probed with centromeric sequence ZmCR2.6c (green). Arrowheads and arrows in $\mathbf{A}$ indicate two types of centromeres (see text). C MI/AI chromosomes (red) probed with ZmCR2.6c (green). Note the presence of signals in stretching regions of the chromosomes. D The same chromosomes in A stained with Giemsa. Arrowheads and arrows indicate two types of centromeres. E Late pachytene chromosomes (red) probed with the 180-bp knob repeat (green). Weak signals are indicated by arrows. Chromosomes are identified from comparison with $\mathbf{B}$ and $\mathbf{F}$. F The same chromosomes (red) as in $\mathbf{B}$ reprobed with the 180-bp repeat (green). Weak signals at the termini of $1 \mathrm{~S}, 2 \mathrm{~S}, 3 \mathrm{~S}, 6 \mathrm{~L}$ and $8 \mathrm{~S}$, in the secondary constriction on $6 \mathrm{~S}$, and at sites in $1 \mathrm{~L}, 4 \mathrm{~L}$ and $5 \mathrm{~S}$ are indicated by arrows. G Pachytene chromosomes (blue) simultaneously probed with $26 \mathrm{~S}$ rDNA (red) and the 180 -bp repeat (green). Note overlapping of red and green signals (arrow) at the distal end of NOR in 6S. H Portion of a PMC at early pachytene stage, showing the distribution $26 \mathrm{~S}$ rDNA (red) and the 180-bp repeat (green) in the short arm (blue) of chromosome 6. Note overlapping of red and green signals (arrow) in the NOR-secondary constriction. I Pachytene chromosomes (blue) simultaneously probed with the180-bp repeat (green) and the TR-1 repeat (red). Chromosome 4 is identified by the presence of two small clusters of 180-bp repeats (arrows) in the long arm. J Portion of a PMC at pachytene stage, showing the position of 5S rDNA (green) in $2 \mathrm{~L}$ (red). Arrow indicates the centromere. Bar: $10 \mu \mathrm{m}$ 

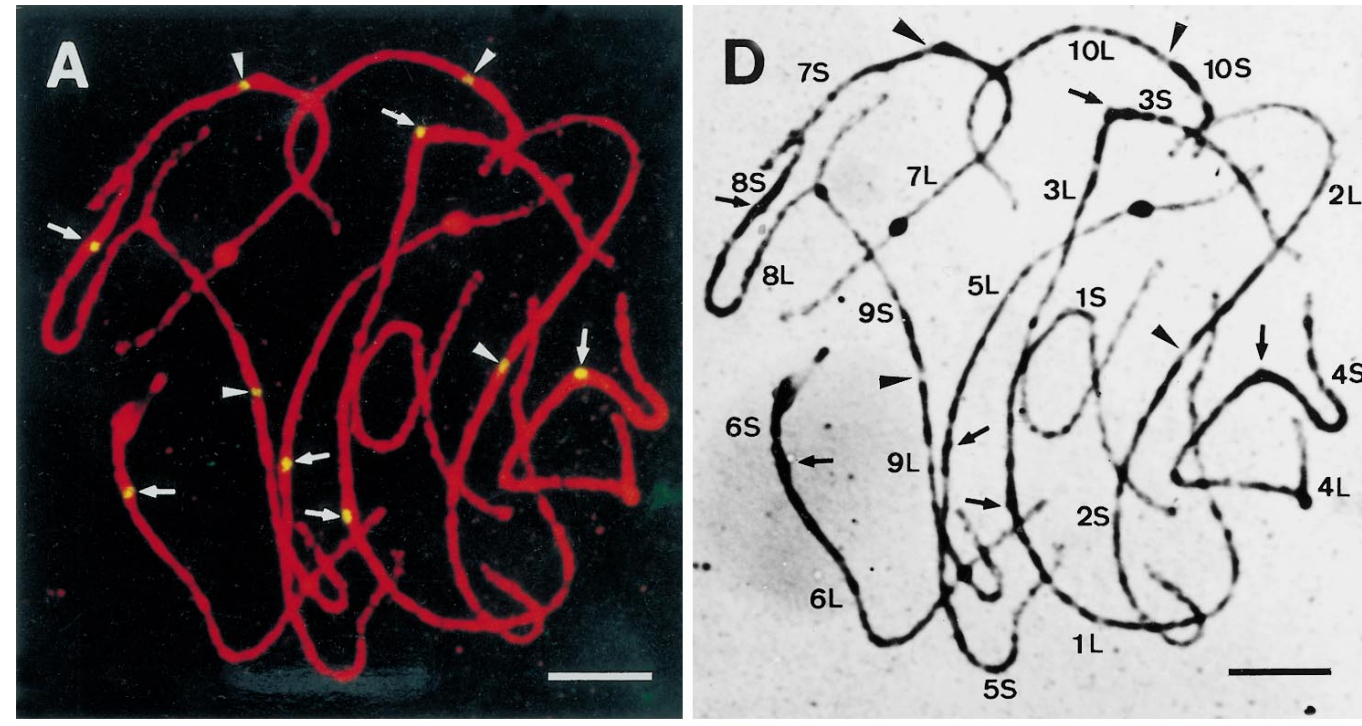

C

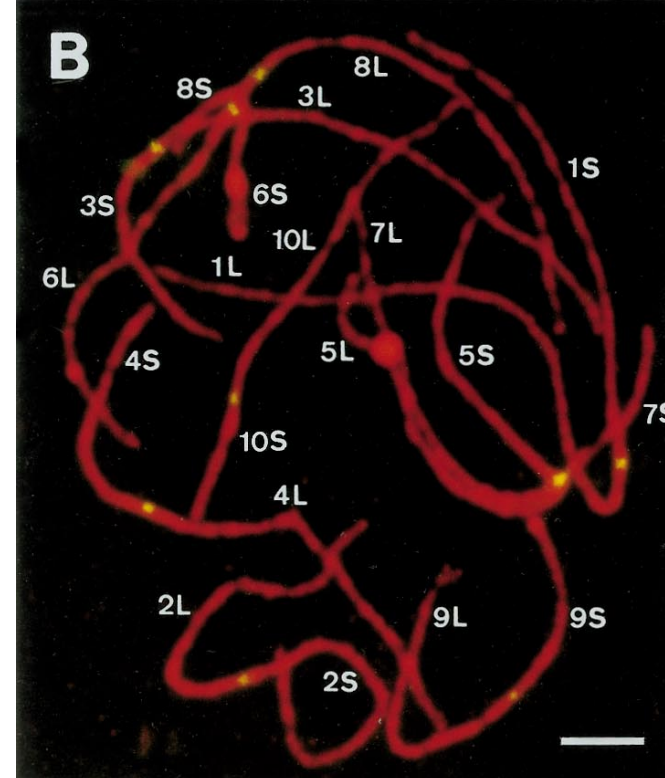

E
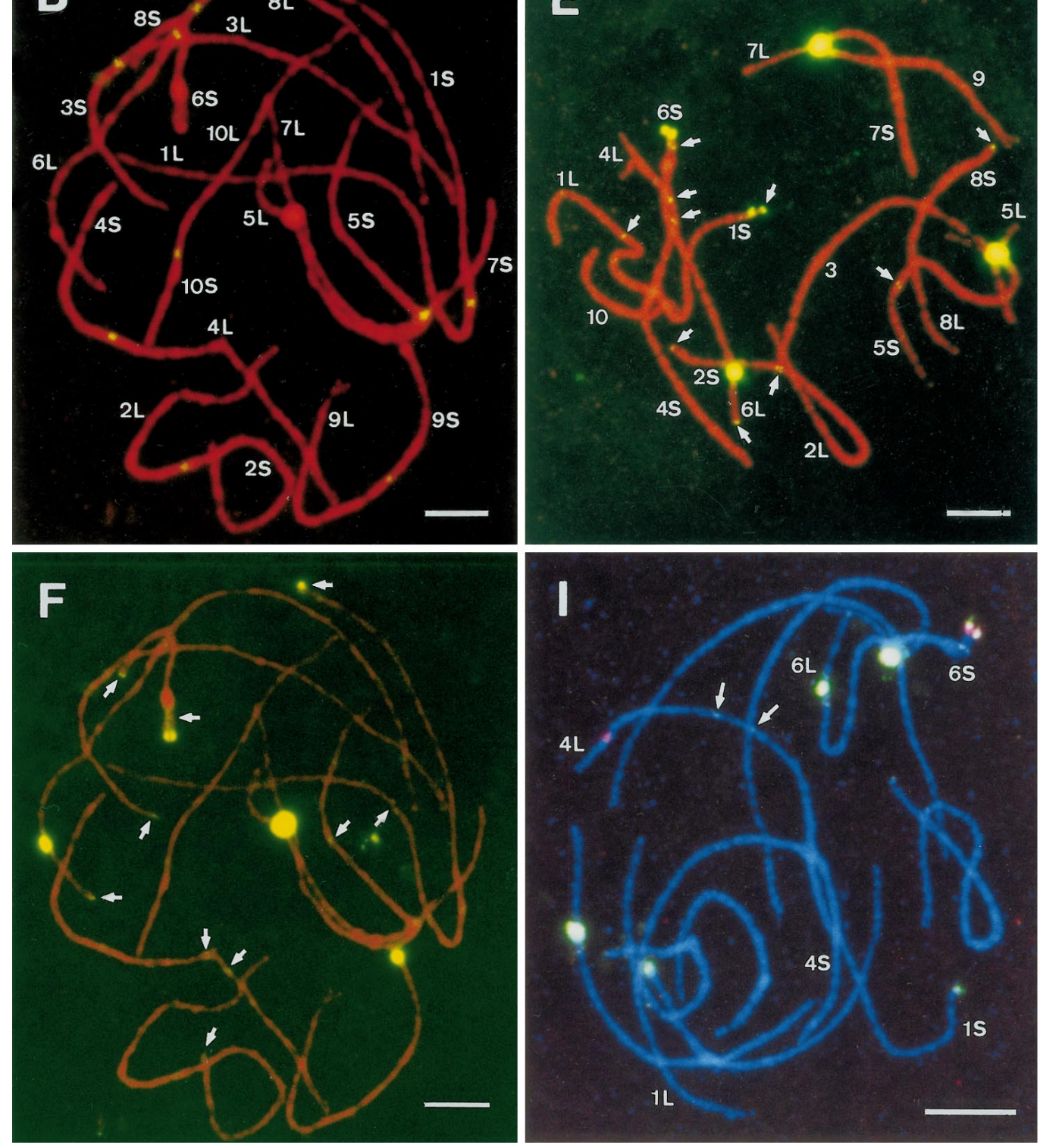

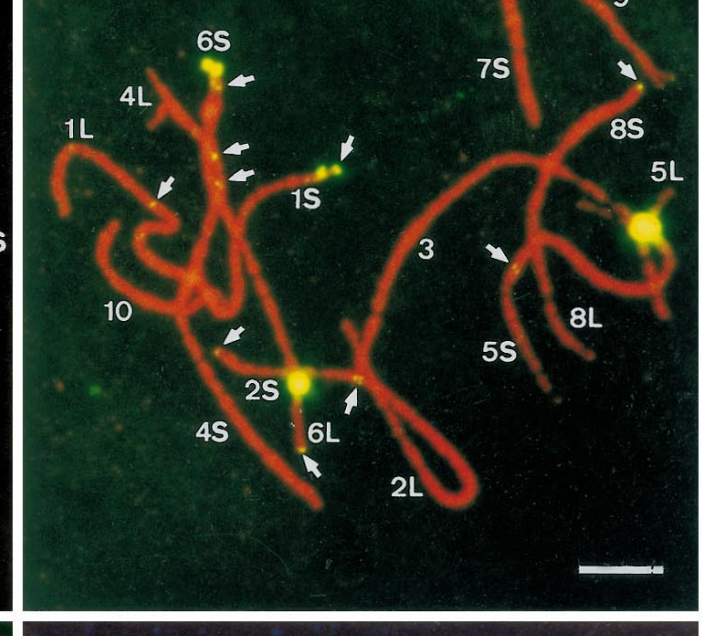

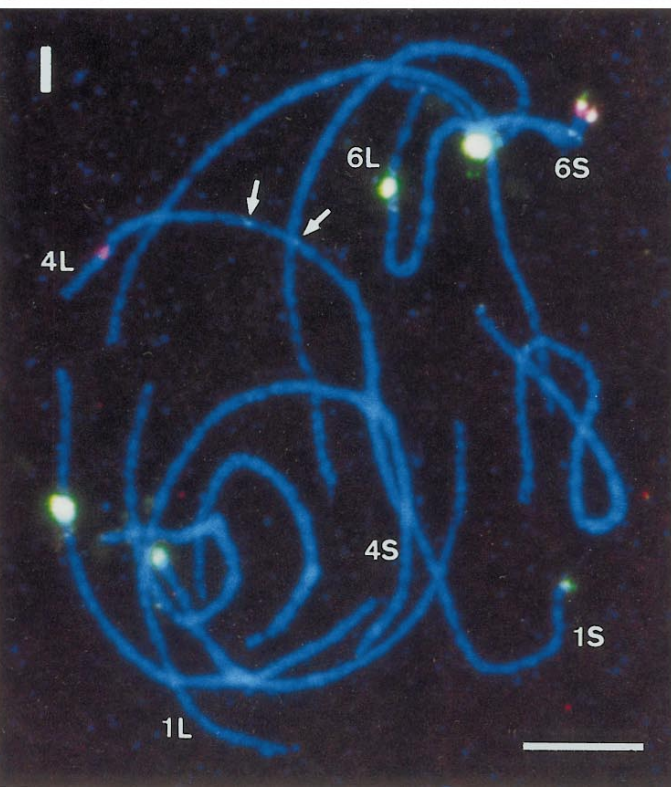

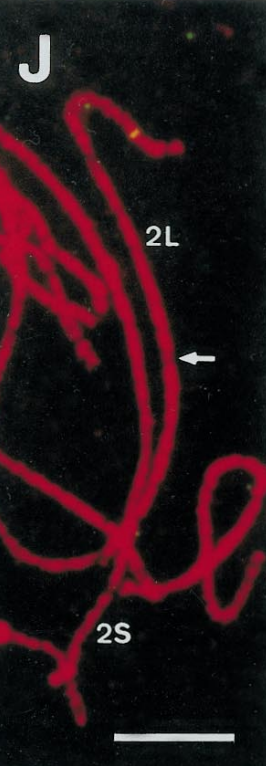

Fig. 1 
Table 1 Lengths and arm ratios of maize pachytene chromosomes derived from the acetocarmine squash technique and FISH

\begin{tabular}{|c|c|c|c|c|c|c|c|c|c|c|}
\hline \multirow{2}{*}{$\begin{array}{l}\text { Chrome- } \\
\text { some }\end{array}$} & \multicolumn{3}{|c|}{ Rhoades 1950} & \multicolumn{2}{|c|}{ Sheridan 1982} & \multicolumn{3}{|c|}{ Neuffer et al. 1997} & \multicolumn{2}{|l|}{ This study } \\
\hline & $\begin{array}{l}\text { Length } \\
(\mu \mathrm{m})\end{array}$ & $\% \mathrm{TCL}^{\mathrm{a}, \mathrm{b}}$ & $\mathrm{L} / \mathrm{S}^{\mathrm{c}}$ & $\% \mathrm{TCL}^{\mathrm{b}}$ & $\mathrm{L} / \mathrm{S}$ & $\begin{array}{l}\text { Unit } \\
\text { Length }\end{array}$ & $\% \mathrm{TCL}^{\mathrm{b}}$ & $\mathrm{L} / \mathrm{S}$ & $\% \mathrm{TCL}$ & $\mathrm{L} / \mathrm{S}$ \\
\hline 1 & 82.40 & 14.91 & 1.30 & 15.66 & 1.46 & 229 & 14.51 & 1.23 & $14.80 \pm 0.64$ & $1.31 \pm 0.13$ \\
\hline 2 & 66.50 & 12.03 & 1.25 & 11.82 & 1.43 & 196 & 12.42 & $1.42,1.14^{\mathrm{e}}$ & $12.27 \pm 0.47$ & $1.17 \pm 0.09$ \\
\hline 3 & 62.00 & 11.22 & 2.00 & 11.04 & 2.32 & 179 & 11.34 & 2.0 & $11.13 \pm 0.55$ & $2.07 \pm 0.13$ \\
\hline 4 & 58.78 & 10.64 & 1.60 & 10.44 & 1.42 & 175 & 11.09 & $1.63,2.0^{\mathrm{e}}$ & $10.96 \pm 0.68$ & $1.67 \pm 0.18$ \\
\hline 5 & 59.82 & 10.82 & 1.10 & 10.69 & 1.14 & 175 & 11.09 & 1.07 & $11.46 \pm 0.76$ & $1.06 \pm 0.09$ \\
\hline 6 & 48.73 & 8.82 & $7.10,3.10^{\mathrm{d}}$ & 8.34 & 2.72 & 122 & 7.73 & 3.1 & $7.93 \pm 0.42$ & $4.33 \pm 0.71$ \\
\hline 7 & 46.78 & 8.46 & 2.80 & 8.79 & 2.60 & 140 & 8.87 & 2.6 & $8.12 \pm 0.48$ & $2.79 \pm 0.40$ \\
\hline 8 & 47.48 & 8.59 & 3.20 & 8.71 & 3.26 & 140 & 8.87 & 3.0 & $8.49 \pm 0.35$ & $3.42 \pm 0.44$ \\
\hline 9 & 43.24 & 7.82 & 1.80 & 7.47 & 2.01 & 122 & 7.73 & 2.0 & $8.08 \pm 0.91$ & $1.93 \pm 0.34$ \\
\hline 10 & 36.93 & 6.68 & 2.80 & 7.04 & 2.70 & 100 & 6.34 & 2.6 & $6.75 \pm 0.56$ & $2.64 \pm 0.15$ \\
\hline
\end{tabular}

a TCL, Total complement length d Dempsey (1994)

${ }^{b}$ Calculated by authors of this paper

e Two forms of chromosomes

c L/S, Long-to-short chromosome arm ratio

Fig. 2A, B Idiogram of the pachytene chromosomes of maize inbred line KYS based on the distribution of repetitive DNA sequences. A Complete chromosome set. B Enlarged portion of $6 \mathrm{~S}$ showing NOR-heterochromatin, NORsecondary constriction, satellite, and terminal knob. Arrows indicate centromeres
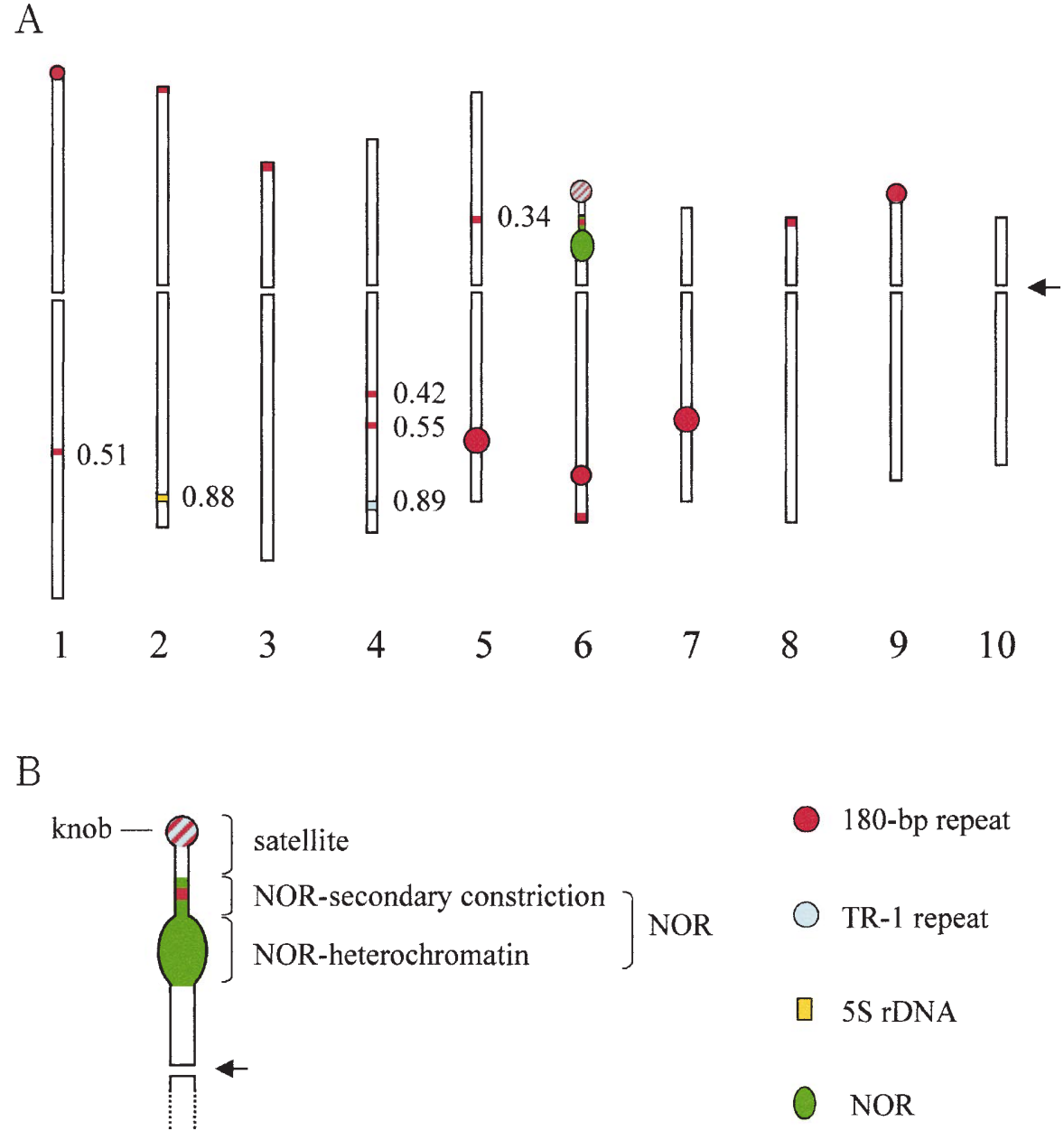

pachynema, when chromosomes were more condensed, signals from 180-bp repeats appeared at the most distal end of rDNA tandem repeats (Fig. 1G). At early pachynema, when chromosomes were less condensed, 180-bp repeats clustered within the NOR-secondary constriction (Fig. 1H).

\section{Localization of TR-1 elements}

Among the many TR-1 element-containing clones isolated, only two were sequenced. Homology among these two clones and one clone isolated by Ananiev et al. (1998b) is over $85 \%$. 
Chromosomal locations of TR-1 elements relative to $180-b p$ repeats were investigated by two-color FISH. In all of the PMCs examined, only two clusters of signals from TR-1 repeats were observed, one located in the long arm of chromosome 4 at a site approximately 0.89 distal to the centromere and the other co-located with signals from 180-bp repeats in the terminal knob (satellite) on 6S (Fig. 1I).

\section{Localization of 5S rDNA}

FISH of rice 5S rDNA to pachytene chromosomes (Fig. 1J) is consistent with the result from radioactive in situ hybridization that 5S rRNA genes are clustered at 2L.88 (Mascia et al. 1981).

\section{Discussion}

CCS1 and pSau3A9 are two centromere-associated sequences originally derived from Brachypodium sylvaticum (Argón-Alcaide et al. 1996) and Sorghum bicolor (Jiang et al. 1996), respectively. Sequences homologous to CCS1 and pSau3A9 have been isolated from other species of the grass family and subsequently mapped to the centromeric regions of somatic metaphase chromosomes by FISH (Ananiev et al. 1998c; Miller et al. 1998; Presting et al. 1998; Nonomura and Kurata 1999). In this study we isolated a maize homology of the sorghum pSau3A9 sequence, ZmCR2.6c, and hybridized it to meiotic chromosomes of maize. Our results clearly indicate that hybridization signals are present in the primary constriction of pachytene bivalents and in the stretched regions of MI and AI chromosomes where the spindle attaches. These results strongly suggest that $\mathrm{ZmCR} 2.6 \mathrm{c}$ is a centromeric sequence, although its function is not known.

Dempsey (1994) recognized two types of centromeres in acetocarmine-stained pachytene spreads of KYS. Centromeres of chromosomes 2, 7, 9, and 10 are large, rectangular in shape, and easily identified, whereas those of the remaining chromosomes are small, circular in shape, and often protrude to one side of the linear axis of the bivalents. Ananiev et al. (1998c) found that two maize centromeric elements, CentA and CentC, differ in proportion and arrangement in different centromeres. In the present investigation, we observed that FISH signals on the first type of centromeres of Dempsey (1994) were weaker, more diffused, and could be distinguished from the surrounding heterochromatin, whereas signals on the second type of centromeres were stronger, more condensed, and embedded in blocks of heterochromatin (Fig. 1A). This may reflect differences in the copy number and arrangement of repetitive and unique sequences in the centromeric regions of different chromosomes.

Since in FISH the pachytene chromosomes are easily traceable and their centromeres can be precisely mapped, we measured chromosome lengths and calculated the arm ratios of several good-quality cells. Our data agree well with those from traditional analysis, except for the arm ratio of chromosome 6 (Table 1). This discrepancy may be attributed to different views on the length of the short arm which carries NOR-heterochromatin, the NOR-secondary constriction, and a satellite.

In this study we have shown by FISH that 180 -bp repeats are present not only in knobs on chromosome arms $5 \mathrm{~L}, 6 \mathrm{~S}, 6 \mathrm{~L}, 7 \mathrm{~L}$, and $9 \mathrm{~S}$ as previously reported (Peacock et al. 1981) but also in regions where heterochromatin is not apparent, such as the termini of $1 \mathrm{~S}, 2 \mathrm{~S}, 3 \mathrm{~S}, 6 \mathrm{~L}$, and $8 \mathrm{~S}$, the NOR-secondary constriction on $6 \mathrm{~S}$, and interstitial regions in $1 \mathrm{~L}, 4 \mathrm{~L}$, and $5 \mathrm{~S}$. This is consistent with results from Southern blot hybridization of the 180-bp repeat to genomic DNA from oat-maize monosomic addition lines (Ananiev et al. 1998a). These investigators found that every Seneca 60 maize chromosome in the lines analyzed possessed 180-bp repeats, with copy numbers ranging from 100 to 25,000 . The abundance, tandem repetitiveness, and chromosome-specific distribution of the 180-bp repeat make it an excellent cytological marker for maize pachytene chromosomes.

The minor 180-bp FISH signals on the termini of $1 \mathrm{~S}$, $2 \mathrm{~S}, 3 \mathrm{~S}$, and at $1 \mathrm{~L} .51$ correspond approximately to the knob positions observed in different races of maize, whereas signals on the termini of $6 \mathrm{~L}, 8 \mathrm{~S}$, and at $4 \mathrm{~L} .42$, 4L.55, and 5S.34 have not been reported to be knob sites (McClintock et al. 1981; Neuffer et al. 1997). The presence of 180-bp repeats in the NOR-secondary constriction observed in this study is inconsistent with the result of Peacock et al. (1981), who found that ${ }^{3} \mathrm{H}$-labeled cRNA of the 180-bp repeat could not hybridize to NOR. Recently, Ananiev et al. (1998b) reported the observation of "a small cluster of signals at the chromomere flanking the NOR on the satellite of chromosome 6" in FISH experiments in which pachytene chromosomes of Seneca 60 were probed with 180 -bp and TR-1 repeats. On the basis of the photographs they presented, we presume that the site of signals is in the NOR-secondary constriction. The presence of 180-bp and TR-1 repeats in the NOR-secondary constriction is novel and worth further investigation.

Recently, Ananiev et al. (1998b) isolated a new type of tandemly repeated sequence, TR-1 elements, from the knob DNA of Seneca 60. TR-1 elements are about $350 \mathrm{bp}$ in length and comprise approximately $2 \%$ of the total genome, an amount equivalent to or not less than that of 180-bp repeats (Ananiev et al. 1998a). Comparative FISH of these two types of repeats indicates eight sites of TR-1 elements on the pachytene chromosome of Seneca 60; of these, five also contain 180-bp repeats (Ananiev et al. 1998b). Peacock et al. (1981) believe that all knobs in KYS are composed of only 180-bp repeats. However, in this study we have isolated TR-1 repeats from genomic DNA of KYS and mapped them to the terminal knob on 6S, where 180-bp repeats are also present, and to a site in $4 \mathrm{~L}$. The co-localization of the two types of repeats in the terminal knob on $6 \mathrm{~S}$ explains why the size of this knob does not correspond with the content of 
180-bp repeats as observed by Peacock et al. (1981). It seems that maize has two types of knob-associated sequences, the 180-bp and TR-1 repeats, which differ in absolute content, relative proportion, and chromosome location among strains. Ananiev et al. (1998b) found two homologous regions between these two types of repeats and proposed that the TR-1 element evolved from 180bp repeat as the result of a duplication and subsequent divergence. These repeats, therefore, are useful tools for investigating the phylogeny and evolution of maize and its relatives.

In this study, we have successfully mapped a centromeric sequence and several tandem repeats to the pachytene chromosomes of maize by FISH (Fig. 2). The combination of chromosome length, centromere position, and distribution of the tandem repeats enabled all chromosomes to be identified with great confidence. Consequently, the technique and results presented form an important basis for mapping low-copy number and unique sequences on chromosomes and allow the integration of the genetic, molecular, and cytological maps of maize.

Acknowledgements We are grateful to Dr. H.K. Wu for his generous gift of the clone pRTy5S and to the Maize Genetics Cooperation Stock Center and Dr. D.B. Walden for supplying seeds of maize inbred line KYS. This study was supported by grant nos. NSC88-2311-B-002-001 and NSC89-2311-B-002-005 from the National Science Council, Republic of China. The experiments performed in this study comply with the current laws of the Republic of China.

\section{References}

Ananiev EV, Phillips RL, Rines HW (1998a) Complex structure of knob DNA on maize chromosome 9: retrotransposon invasion into heterochromatin. Genetics 149:2025-2037

Ananiev EV, Phillips RL, Rines HW (1998b) A knob-associated tandem repeat in maize capable of forming fold-back DNA segments: are chromosome knobs megatransposons? Proc Natl Acad Sci USA 95:10785-10790

Ananiev EV, Phillips RL, Rines HW (1998c) Chromosome-specific molecular organization of maize (Zea mays L.) centromeric regions. Proc Natl Acad Sci USA 95:13073-13078

Aragón-Alcaide L, Miller T, Schwarzacher T, Reader S, Moore G (1996) A cereal centromeric sequence. Chromosoma 105:261268

Armstrong SJ, Fransz P, Marshall DF, Jones GH (1998) Physical mapping of DNA repetitive sequences to mitotic and meiotic chromosomes of Brassica oleracea var. alboglabra by fluorescence in situ hybridization. Heredity 81:666-673

Carlson WR (1988) The cytogenetics of corn. In: Sprague GF, Dudley JW (eds) Corn and corn improvement. American Society of Agronomy, Madison, Wis., pp 259-344

Chen CC, Chen CM, Yang JT, Kao YY (1998) Localization of a repetitive DNA sequence to the primary constrictions of maize pachytene chromosomes. Chromosome Res 6:236-238

Chung MC, Ning CN, Wu HK (1993) Localization of ribosomal RNA genes on rice chromosomes. Bot Bull Acad Sin 34:4355

Dempsey E (1994) Traditional analysis of maize pachytene chromosomes. In: Freeling M, Walbot V (eds) The maize handbook. Springer, Berlin Heidelberg New York, pp 432-441

Dennis ES, Peacock WJ (1984) Knob heterochromatin homology in maize and its relatives. J Mol Evol 20:341-345
Dong F, Miller JT, Jackson SA, Wang GL, Ronald PC, Jiang J (1998) Rice (Oryza sativa) centromeric regions consist of complex DNA. Proc Natl Acad Sci USA 95:8135-8140

Edwards KJ, Thompson H, Edwards D, de Saizien A, Sparks C, Thompson JA, Greenland AJ, Eyers M, Schuch W (1992) Construction and characterisation of a yeast artificial chromosome library containing three haploid maize genome equivalents. Plant Mol Biol 19:299-308

Fransz P, Armstrong S, Alonso-Blanco C, Fischer TC, Torres-Ruiz RA, Jones G (1998) Cytogenetics for the model system Arabidopsis thaliana. Plant J 13:867-876

Heslop-Harrison JS, Harrison GE, Leitch IJ (1992) Reprobing of DNA:DNA in situ hybridization preparations. Trends Genet 8:372-373

Jiang J, Gill BS (1994) Nonisotopic in situ hybridization and plant genome mapping: the first 10 years. Genome 37:717-725

Jiang J, Nasuda S, Dong F, Scherrer CW, Woo SS, Wing RA, Gill BS (1996) A conserved repetitive DNA element located in the centromeres of cereal chromosomes. Proc Natl Acad Sci USA 93:14210-14213

Mascia PN, Rubenstein I, Phillips RL, Wang AS, Xiang LZ (1981) Localization of the 5S rRNA genes and evidence for diversity in the 5S rDNA region of maize. Gene 15:7-20

McClintock B, Kato TA, Blumenschein A (1981) Chromosome constitution of races of maize. Colego de Postgraduados Chapingo, Mexico

Miller JT, Dong F, Jackson SA, Song J, Jiang J (1998) Retrotransposon-related DNA sequences in the centromeres of grass chromosomes. Genetics 150:1615-1623

Neuffer MG, Coe EH, Wessler SR (1997) Mutants of maize. Cold Spring Harbor Laboratory Press, New York

Nonomura KI, Kurata N (1999) Organization of the 1.9-kb repeat unit RCE1 in the centromeric region of rice chromosomes. Mol Gen Genet 261:1-10

Peacock WJ, Dennis ES, Rhoades MM, Pryor AJ (1981) Highly repeated DNA sequence limited to knob heterochromatin. Proc Natl Acad Sci USA 78:4490-4494

Presting GG, Malysheva L, Fuchs J, Schubert I (1998) A Ty3/gypsy retrotransposon-like sequence localizes to the centromeric regions of cereal chromosomes. Plant J 16:721-728

Rhoades MM (1950) Meiosis in maize. J Hered 41:58-67

Sambrook J, Fritsch EF, Maniatis T (1989) Molecular cloning: a laboratory manual. Cold Spring Harbor Laboratory Press, New York

Sheridan WF (1982) Maps, markers and stocks. In: Sheridan WF (ed) Maize for biological research. Plant Mol Biol Assoc, Charlottesville, Va., pp 37-52

Suen DF, Wang CK, Lin RF, Kao YY, Lee FM, Chen CC (1997) Assignment of DNA markers to Nicotiana sylvestris chromosomes using monosomic alien addition lines. Theor Appl Genet 94:331-337

Trask BJ (1991) Fluorescence in situ hybridization: applications in cytogenetics and gene mapping. Trends Genet 5:149-154

Viegas-Péquignot E, Berrard S, Brice A, Apion F, Mallet J (1991) Localization of a 900-bp-long fragment of the human choline acetyltransferase gene to $10 \mathrm{q} 11.2$ by nonradioactive in situ hybridization. Genomics 9:210-212

Xu J, Earle ED (1996a) Direct FISH of 5S rDNA on tomato pachytene chromosomes places the gene at the heterochromatic knob immediately adjacent to the centromere of chromosome 1. Genome 39:216-221

$\mathrm{Xu} \mathrm{J}$, Earle ED (1996b) High resolution physical mapping of $45 \mathrm{~S}(5.8 \mathrm{~S}, 18 \mathrm{~S}$ and $25 \mathrm{~S})$ rDNA gene loci in the tomato genome using a combination of karyotyping and FISH of pachytene chromosomes. Chromosoma 104:545-550

Zhong X, de Jong JH, Zabel P (1996a) Localization of repetitive sequences on metaphase and pachytene chromosomes of tomato using fluorescence in situ hybridization. Chromosome Res 4:24-28

Zhong X, Fransz PF, Wennekes-van Eden J, Zabel P, Van Kammen A, de Jong JH (1996b) High resolution mapping on pachytene chromosomes and extended DNA fibres by fluorescence in situ hybridization. Plant Mol Biol Rep 14:232-242 